\title{
The Influence of Sea Surface Temperatures on the Northern Winter Stratosphere: Ensemble Simulations with the MAECHAM5 Model
}

\author{
E. MANZINI \\ National Institute for Geophysics and Volcanology, Bologna, Italy \\ M. A. Giorgetta, M. Esch, L. Kornblueh, And E. Roeckner \\ Max Planck Institute for Meteorology, Hamburg, Germany
}

(Manuscript received 10 November 2004, in final form 26 August 2005)

\begin{abstract}
The role of interannual variations in sea surface temperatures (SSTs) on the Northern Hemisphere winter polar stratospheric circulation is addressed by means of an ensemble of nine simulations performed with the middle atmosphere configuration of the ECHAM5 model forced with observed SSTs during the 20-yr period from 1980 to 1999. Results are compared to the 40-yr ECMWF Re-Analysis (ERA-40). Three aspects have been considered: the influence of the interannual SST variations on the climatological mean state, the response to El Niño-Southern Oscillation (ENSO) events, and the influence on systematic temperature changes. The strongest influence of SST variations has been found for the warm ENSO events considered. Namely, it has been found that the large-scale pattern associated with the extratropical tropospheric response to the ENSO phenomenon during northern winter enhances the forcing and the vertical propagation into the stratosphere of the quasi-stationary planetary waves emerging from the troposphere. This enhanced planetary wave disturbance thereafter results in a polar warming of a few degrees in the lower stratosphere in late winter and early spring. Consequently, the polar vortex is weakened, and the warm ENSO influence clearly emerges also in the zonal-mean flow. In contrast, the cold ENSO events considered do not appear to have an influence distinguishable from that of internal variability. It is also not straightforward to deduce the influence of the SSTs on the climatological mean state from the simulations performed, because the simulated internal variability of the stratosphere is large, a realistic feature. Moreover, the results of the ensemble of simulations provide weak to negligible evidence for the possibility that SST variations during the two decades considered are substantially contributing to changes in the polar temperature in the winter lower stratosphere.
\end{abstract}

\section{Introduction}

In the Northern Hemisphere, the winter stratosphere is known to be characterized by variations in the strength and structure of the polar vortex covering a wide range of time scales. On short time scales (days and weeks) disturbances of the stratospheric polar vortex are associated with sudden stratospheric warming events [see, among many others, Labitzke (1981), Matsuno (1971), and Andrews et al. (1987); for a recent analysis, see Limpasuvan et al. (2004)]. There is clear evidence, reported below, that on short time scales the

Corresponding author address: E. Manzini, National Institute for Geophysics and Volcanology, Via Donato Creti 12, 40128 Bologna, Italy.

E-mail: manzini@bo.ingv.it polar vortex variability may be intrinsically generated within the atmosphere, or even the stratosphere itself. However, it still is an open question how much natural and anthropogenic external factors determine a particular history of variations of the stratospheric polar vortex on relatively long time scales, namely, from weeks to a season, on interannual and on decadal time scales.

From interannual to interdecadal time scales, possible natural factors of influence on the polar vortex variability include the quasi-biennial oscillation (QBO) in equatorial zonal wind, the semiannual oscillation (SAO) in equatorial zonal wind at the stratopause, changes associated with solar irradiance variations, volcanic effects, and changes associated with variations in sea surface temperature (SST). Anthropogenic factors include changes in ozone-depleting substances and, 
consequently, ozone changes, and changes in greenhouse gases. Note that changes in SST contain also part of the signal from atmospheric composition changes, such as the increase in greenhouse gases.

The purpose of the current work is to evaluate the role of observed SST interannual variations on the Northern Hemisphere winter stratosphere by analyzing ensemble simulations and reanalysis data for the 198099 period. The model used to perform the ensemble of simulations is the newly configured middle atmosphere version of the most recent ECHAM model suite (MAECHAM5) general circulation model (Roeckner et al. 2003, 2006). A side purpose of this work is, therefore, to introduce the newly configured MAECHAM5 model. For comparison and validation of the model results, the 40-yr European Centre for Medium-Range Weather Forecasts Re-Analysis (ERA-40) is used. We consider the evaluation of the influence of SST variations on the polar vortex with a GCM, a necessary step in the larger context of establishing confidence in the role of dynamical processes in ozone predictions for the future decades with chemistry climate models in general and, specifically, those models that will be based on the MAECHAM5 GCM.

Early observational works (van Loon and Labitzke 1987; Hamilton 1993a; Baldwin and O'Sullivan 1995; Kodera et al. 1996) dealing with the influence of SST variations on the northern winter stratospheric polar vortex have provided evidence for an enhancement of the wave character of the lower stratosphere, namely an intensification of the Aleutian high, during El NiñoSouthern Oscillation (ENSO) events. These works also pointed out the difficulty in distinguishing between ENSO and QBO effects on the northern polar vortex and did not give conclusive results concerning an ENSO influence on the zonal-mean circulation of the extratropical stratosphere. Part of this picture is accepted currently (Calvo Fernandez et al. 2004).

Concerning the ability of middle atmosphere general circulation models to simulate stratospheric variability, it is summarized that models including a comprehensive representation of the troposphere, relatively modest horizontal and vertical resolutions, climatological boundary conditions, and no variations in external forcing have been shown to be generally successful in representing the fundamental aspects of the polar vortex and its perturbations. For instance, the occasional occurrence of sudden stratospheric warming events has been reported in many models (Boville 1995; Hamilton et al. 1995; Manzini and Bengtsson 1996; Lahoz 2000: among others).

Simulations of the middle atmosphere with general circulation models have therefore demonstrated that the variability of the polar vortex may arise spontaneously from nonlinear atmospheric dynamics. This type of variability is commonly referred to as internal variability. Focusing on the polar vortex in the stratosphere, a further distinction can be made, namely, that stratospheric variability can arise as a response to the internal variability of the troposphere or being, instead, generated internally by the stratosphere itself. From simulations with a general circulation model that includes realistic representations of both the troposphere and the stratosphere it is not straightforward to distinguish between these two types of stratospheric variability. However, simplified modeling specifically aimed at such questions has provided evidence of both (Matsuno 1971; Holton and Mass 1976; Kodera and Kuroda 2000; Gray 2003).

Previous modeling work dealing with the influence of SST on the polar stratosphere includes simulations with idealized SST anomalies (Hamilton 1993b), an ensemble of nine simulations covering the northern winter season (Lahoz 2000), comparisons between simulations with and without SST variations (Braesicke and Pyle 2004), and an ensemble of three simulations covering the last 50 years (Sassi et al. 2004). All of them considered only SST variations and the models used did not spontaneously simulate a QBO. Sassi et al. (2004) aimed at extracting the variability associated with ENSO events and reported a more disturbed polar stratosphere for warm ENSO events. However the work of Lahoz (2000) did not. Moreover, the SST influence reported by Braesicke and Pyle (2004) does not appear to be related to ENSO variability. Therefore, concerning ENSO influence on the northern stratosphere in winter, model simulations do not yet deliver conclusive results. Lahoz (2000), in addition, addressed the issue of temperature trends in the northern polar stratosphere and inferred from his ensemble of seasonal simulations that the SST variations from the 1980 s to the 1990 s can explain at least in part the observed cooling in the stratosphere.

For the analysis presented in this work, an ensemble of nine simulations with the MAECHAM5 model has been performed. Each simulation ranges over 20 years and is forced with observed monthly mean SST and sea ice concentration (SIC) from 1980 to 1999, and differs only for the initial conditions.

This experimental design is therefore an extension of both Lahoz (2000), given that it considers continuous simulations instead of seasonal simulations, and Sassi et al. (2004), given that it considers a much larger number of total realizations. Although a shorter time span has been used here in comparison with Sassi et al. (2004), the availability of more realizations should lead to the 
construction of more homogeneous ENSO composite maps and, therefore, help in providing unambiguous evidence for the ENSO influence. The availability of more realizations indeed allows for the separate composite analysis of ENSO events and their cold counterparts so that distortions in the interpretation of the midlatitude tropospheric response to ENSO are avoided (Hoerling et al. 1997; Strauss and Shukla 2002).

Similarly to the reported previous works, the MAECHAM5 model employed here does not spontaneously simulate the QBO; therefore, as for the previous modeling works, it is possible to focus on the ENSO influence alone. In particular, the focus of the current investigation is on the stratospheric response to ENSOinduced modifications of the extratropical circulation in the troposphere, although other forms of influence, associated for instance with the influence of ENSO on the tropical stratosphere, cannot be ruled out.

Since the MAECHAM5 experimental design is also an extension of Lahoz (2000), we can also address the question of whether the ensemble simulations with the MAECHAM5 model confirm his results concerning temperature trends in the lower stratosphere. For comparison with Braesicke and Pyle (2004) and addressing the issue of the SST influence on the climatological mean, an additional 20-yr simulation with climatological SST has been used.

An important aspect of this work is also to compare the MAECHAM5 model results to a consistent analysis of 20 years of ERA-40 data (Uppala et al. 2004), one of the best estimates of the observed atmospheric state and its evolution. In the comparison between the model and the reanalysis, it is important to consider that in nature only one time evolution of the atmospheric state is realized. Therefore, it is expected for the ensemble mean results to be smoother than the reanalysis, because the ensemble mean is the average of a number of realizations, and therefore the internal variability has been filtered out to a larger extent than in composites and/or time means derived from the analysis data. For the same reasons, care must be taken in the comparison of the magnitudes of the anomalies. The point of interest is whether it is possible to identify common structure and behaviors, possibly characterizing the atmospheric response to the considered external forcing, in this case the SSTs.

In summary, the model and the approach used in the analysis of the results are presented in section 2 . The reanalysis used for comparison and validation of the model results are introduced in section 3 . Three aspects of SST influence are respectively considered in section 4 , dealing with the SST influence on the climatological mean; in section 5, focusing on the ENSO influence; and in section 6, addressing the SST influence on temperature trends. General conclusions and implications for future works are drawn in section 7 .

\section{Model and methodology}

\section{a. Model}

MAECHAM5 is the middle atmosphere configuration of the ECHAM5 model presented by Roeckner et al. (2006) and detailed in Roeckner et al. (2003). It therefore includes all the novel features discussed by Roeckner et al. (2003). Given the low vertical resolution employed (the standard resolution used also in the previous model cycle, MAECHAM4), the QBO does not spontaneously occur in the model simulations considered here (Giorgetta et al. 2002). The MAECHAM5 model is used at T42 horizontal truncation and with 39 vertical layers.

Concerning gravity wave parameterizations, two modifications are reported with respect to the MAECHAM4 model. 1) The orographic scheme has been changed; the current model uses the Lott and Miller (1997) parameterization (see Roeckner et al. 2003, 2006). Therefore, both the ECHAM5 and the MAECHAM5 models use the same orographic scheme. However, in MAECHAM5 the orographic gravity waves may also break and dissipate at elevations above $10 \mathrm{hPa}$, which are not resolved in tropospheric ECHAM5. 2) The specification of the gravity wave source spectrum of the Hines parameterization (of the momentum flux deposition from an atmospheric gravity wave spectrum) has been changed; the current model uses space- and time-constant gravity wave parameters for the source spectrum, as in case UNI2 of Charron and Manzini (2002). Therefore, an isotropic spectrum with gravity wave wind speed of $1 \mathrm{~m} \mathrm{~s}^{-1}$ and effective horizontal wavenumber $K^{*}=2 \pi(126 \mathrm{~km})^{-1}$ is launched from the lower troposphere, at about $600 \mathrm{hPa}$.

For a basic description of the Hines parameterization, as implemented in the MAECHAM model suite, and the sensitivity of the simulated middle atmosphere to the source spectrum see Manzini et al. (1997), Manzini and McFarlane (1998), and Charron and Manzini (2002). The Hines parameterization is active only in the middle atmosphere model versions, as well as in the Hamburg Model for the Neutral and Ionized Atmosphere (HAMMONIA; Schmidt et al. 2006).

\section{b. Methodology}

An ensemble of nine simulations with observed monthly mean SST and SIC [Atmospheric Model Intercomparison Project II (AMIP II) dataset, Program 
for Climate Model Diagnosis and Intercomparison, available online at http://www-pcmdi.llnl.gov/projects/ amip/AMIP2EXPDSN/BCS/amip2bcs.html] has been performed with the MAECHAM5 model. Each simulation covers 20 years after spinup and differs only for the initial conditions, taken from a 20 -yr control simulation with climatological SST and SIC distribution. Greenhouse gases are held fixed at present values, and a monthly zonal-mean climatological ozone distribution (Fortuin and Kelder 1998) is used, consistent with the simulations for present climate done with the ECHAM5 model. Results are presented for the 20 years with boundary forcing for the 1980-99 period. In addition, results from the $20-y r$ simulation with the 1980-99 climatological SST and SIC are considered. This latter simulation is denoted "climSST."

Given the ensemble of simulations, the evaluation of the role of SST interannual variations on the northern winter stratosphere is based on the 9 (members) $\times 20$ (yr) monthly means of fundamental meteorological variables. The statistical analysis used follows the approach of Strauss and Shukla $(2000,2002)$ and Rowell et al. (1995).

The ensemble mean is defined to be the 20-yr time series of the monthly means obtained by averaging the nine members of the ensemble, for each month of the years considered. The influence of the SST and SIC distributions is contained in the ensemble mean time series. The climatological mean of each calendar month is computed in the usual way from the 20-yr ensemble mean time series.

For each calendar month, the variance and standard deviation of the ensemble mean time series with respect to the climatological mean are defined to be the ensemble variance and ensemble standard deviation, respectively. They are a measure of the atmospheric variability associated with the external forcing, in this case the SST and SIC distributions. Given that a finite ensemble size must be considered, the ensemble mean and its variance are still contaminated by internal variability.

The information concerning internal atmospheric variability is contained in the internal variance, derived from the ensemble of the monthly mean deviations from the ensemble mean time series.

The total variance is the unbiased sum of the ensemble and internal variances, in order to take into account the finite size of the ensemble (Strauss and Shukla 2000).

For the computation of the statistical significance of the difference between the two decades considered (1990s minus 1980s, section 6), the ensemble, internal,
TABLE 1. Warm and cold ENSO and NEUTRAL years (year index for January).

\begin{tabular}{ll}
\hline \hline Warm ENSO & $1983,1987,1992,1998$ \\
Cold ENSO & $1985,1989,1996,1999$ \\
NEUTRAL & $1981,1982,1984,1986,1988,1990,1991,1993$, \\
& $1994,1995,1997$ \\
\hline
\end{tabular}

and total variances are separately recomputed for each of the two decades.

\section{c. ENSO composites}

The SST anomalies in the Niño-3 region from the National Centers for Environmental Prediction (NCEP)/ Climate Prediction Center (CPC) (http://www.cpc.ncep. noaa.gov/data/indices) have been used to construct the composites for the period 1980-99. The four warmest SST Niño-3 anomalies averaged from January to March have been denoted warm ENSO events and the four coldest anomalies, cold ENSO events. The same warm and cold ENSO events are obtained using Niño-3.4 temperature anomalies. The remaining 11 years have been classified as NEUTRAL (note that 1980 has been excluded because the 1979 fall and winter months are not part of the simulations). The resulting list (Table 1) reproduces the known classifications of ENSO events reported in the literature (e.g., Hoerling et al. 1997; Strauss and Shukla 2002; Calvo Fernandez et al. 2004).

Composites of monthly means have been constructed from the ensemble mean time series, respectively including only the warm ENSO, cold ENSO, and NEUTRAL years. Time series of the composites covering the extended winter-spring season ( 7 months), from October (of the previous year) to April are considered. Given the nonlinearity of the tropospheric teleconnections related to ENSO events (Hoerling et al. 1997), anomalies are here defined as the warm ENSO (cold ENSO) composite minus the NEUTRAL composite. Henceforth, these composite differences are respectively denoted wENSO and cENSO anomalies. The ensemble, internal and total variances of the three composites are computed as detailed in the previous subsection, but considering only the years included in each composite, respectively. Therefore, there are 36 (9 members $\times 4 \mathrm{yr})$ cases for either the warm or cold ENSO composite, and 99 ( 9 members $\times 11 \mathrm{yr}$ ) cases for the NEUTRAL composite.

\section{Reanalysis}

The 1980-99 time series of monthly mean temperature, geopotential, and zonal winds from the ERA-40 
data has been used to compute climatologial means, standard deviations, and ENSO composites for comparisons with the model results. The ENSO composites are computed in the same way as for the simulation, but for the fact that now the size of the ensemble is of course only one member. An alternative would have been to include more ENSO cases from the reanalysis, given that data back to the beginning of the 1960s are available. However, ENSO is a very irregular phenomenon. Therefore, we preferred to focus only on the strongest cases covered by the simulations.

ERA-40 results are shown only from the surface to $1 \mathrm{hPa}$ (instead of up to $0.1 \mathrm{hPa}$ ) to avoid contamination from the model top boundary. For a detailed presentation of the ERA-40 data see Uppala et al. (2004) (and also http://www.ecmwf.int/research/era). For a discussion of the representation of the stratosphere in ERA40 see Randel et al. (2004).

In addition, the same diagnostics have been derived from the NCEP/CPC analysis. The latter is not presented here and has served only to provide confidence in the ERA-40 results, given that it is known that spurious variability is present in the stratosphere of ERA40 (Randel et al. 2004). For the last 20 years of the reanalysis, spurious variability in the ERA-40 data can be seen, for instance, in the standard deviation of monthly zonal-mean temperature, at polar latitudes in the stratosphere (not shown). These problems appear to be restricted to the last 20 years. In the troposphere and lower stratosphere, it is reassuring to find that the two analyses do present consistent results. However, in the upper stratosphere the ENSO anomalies derived from the ERA-40 data appear to be $20 \%-25 \%$ larger than those derived from the NCEP/CPC. Given that the point of interest in the comparison between the results from the ensemble of simulations and the reanalysis is to identify common structure and behaviors, this large uncertainty in the magnitude of the anomalies, confined to the upper stratosphere, is not considered a major shortcoming.

\section{SST influence on the climatological mean}

The influence of the SST variations on the climatological mean of the zonal-mean zonal wind in shown in Fig. 1a where the difference, climatological mean from the ensemble simulations minus the 20 -yr time average from the climSST simulation, is shown for January. The zonal-mean zonal winds are shown because of their fundamental characterization of the stratospheric flow. For other winter and early spring months, similar results are obtained. Generally, the largest differences are found in the Tropics and in the subtropics. The differences in the Tropics, although they may be of interest, are excluded from the discussion because of the lack of the QBO in zonal winds in all the simulations considered here. It is only commented that these results may suggest a role of the SST on the forcing and variability of the QBO. In this model version, because of the lack of the QBO, such possible influence appears to affect directly the semiannual oscillation (SAO) in zonal winds. Of importance to the polar night stratospheric jet, it is noted that for January (and the other northern winter months, although to different degrees) the difference is positive in the subtropics and midlatitudes, suggesting an equatorward shift of the stratospheric jet core in the Northern Hemisphere. The forcing associated with the SST variations may therefore contribute to the alleviation of a typical middle atmosphere model bias, namely the polar confinement of the stratospheric jet (Pawson et al. 2000). We also note a shift in the tropospheric jet in the Southern Hemisphere; however, this is a topic not covered by this work.

Negative differences at high northern latitudes in January are consistent with the results of Braesicke and Pyle (2004). However, in our case such differences are smaller and not statistically significant. Note that in Fig. 1a shaded areas denote regions where the difference is statistically significant at the $95 \%$ and $99 \%$ level according to the Student's $t$ test. A simple Student's $t$ test can be applied because it has been found that the total variance from the ensemble simulations and that from the climSST simulation are within a factor of 2 in most of the middle and high latitudes, the region of interest here.

For reference, the climatological January zonal-mean zonal winds from the ensemble simulations are also shown in Fig. 1c. The MAECHAM5 January zonalmean zonal winds compare favorably with those from the reanalysis (Fig. 1e).

To characterize the source of variability in the ensemble simulations, the ensemble and internal standard deviations for the January zonal-mean zonal wind are plotted in Figs. 1b and 1d, respectively. Given that the MAECHAM model suite has been previously characterized by a substantial internal variability of the stratosphere (Manzini and Bengtsson 1996; Manzini et al. 2003), it is not surprising that the latest version of the MAECHAM model suite (cycle 5) also presents a fair amount of internal variability (Fig. 1d). Quantitatively, the total variance produced by the ensemble of simulations at middle and high latitudes is comparable to that estimated by ERA-40 (Fig. 1f). Both the ensemble and internal standard deviations tend to be largest at high latitudes and present a broader maximum in latitude in the upper stratosphere, a realistic feature (cf. 


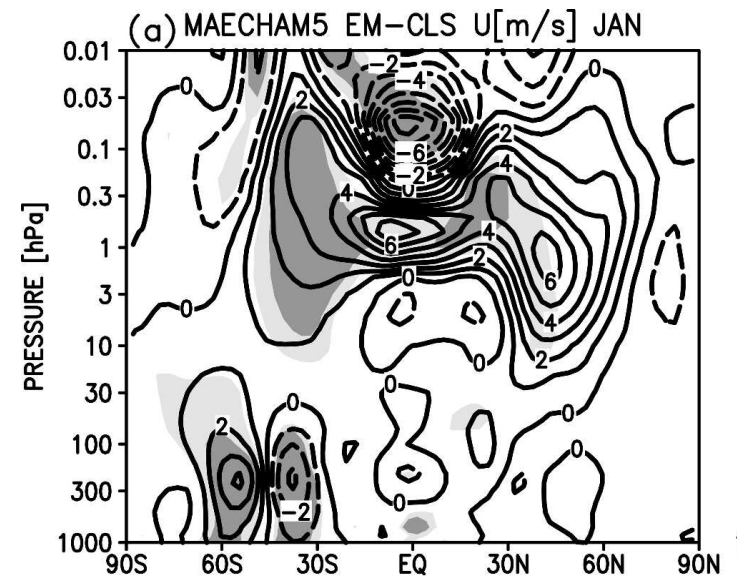

(b) MAECHAM5 EM SD U[m/s] JAN
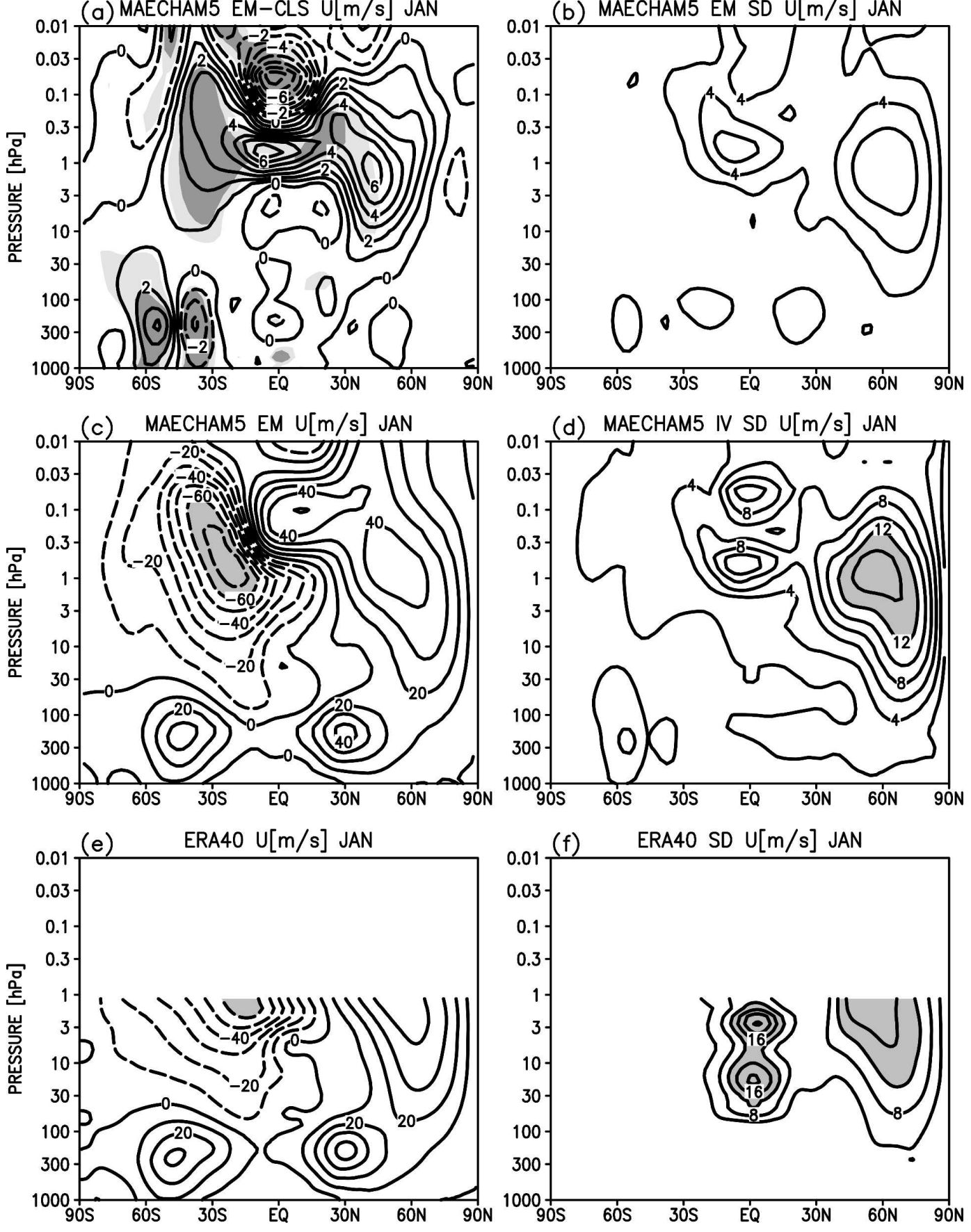

FIG. 1. January zonal-mean zonal wind: (a) the difference (contour: $1 \mathrm{~m} \mathrm{~s}^{-1}$ ) between the climatological means from the ensemble of simulations and from the climSST simulation, statistical significance at the 99\% (95\%) level in dark (light) shade; the climatological mean (c) from the ensemble of simulations and (e) from the ERA-40 (10 $\mathrm{m} \mathrm{s}^{-1}$, values less than $-60 \mathrm{~m} \mathrm{~s}^{-1}$ shaded); (b) ensemble and (d) internal standard deviations from the ensemble of simulations (contour: $2 \mathrm{~m} \mathrm{~s}^{-1}$, values larger then $12 \mathrm{~m} \mathrm{~s}^{-1}$ shaded); (f) the ERA-40 standard deviation (contour: $4 \mathrm{~m} \mathrm{~s}^{-1}$, values larger than $12 \mathrm{~m} \mathrm{~s}^{-1}$ shaded).

with Fig. 1f). The ensemble standard deviation (Fig. 1b) is at most half of the internal (Fig. 1d) standard deviation, reaffirming the dominant role of internal dynamics as a source of stratospheric variability. The standard deviation comparison, therefore, suggests at best a modest contribution of the SST variations to the interannual stratospheric variability on the whole of the two decades considered. 
Note that the simulated maxima of both the ensemble and internal standard deviations in the equatorial mesosphere may be, on the one hand, a realistic feature (associated with variations of the SAO) but, on the other hand, a distorted result because of the lack of the QBO in these simulations. The lack of the QBO is, indeed, the reason for the severe underestimation of the standard deviations in the equatorial lower stratosphere, as demonstrated by Giorgetta et al. (2006).

In summary, it is not straightforward to deduce the influence of the SSTs on the stratospheric mean state from the simulations performed for the MAECHAM5 model. However, this result does not exclude the possibility that the SSTs may influence the stratospheric state on particular occasions and thus affect its time history. This is addressed in the next section considering the ENSO phenomenon.

\section{ENSO influence on the northern winter stratosphere}

The wENSO temperature anomaly for January and February at 5, 50, and $500 \mathrm{hPa}$ from the ensemble of simulations is shown in Fig. 2. The respective wENSO temperature anomaly from the ERA-40 data is shown in Fig. 3. Shading denotes statistical significance at the $95 \%$ and $99 \%$ levels according to the Student's $t$ test. In the troposphere $(500 \mathrm{hPa})$, January and February of both the ERA-40 and model-derived data show positive temperature anomalies over northern America and negative anomalies over the northwestern Pacific Ocean. The negative anomalies extend eastward and southward over the North American continent. This pattern is smooth and significant over large areas for MAECHAM5, given the relatively large number of cases included. As mentioned before, it makes sense that the ERA-40 field is noisier and significant only over relatively small areas. The fact that the results in the troposphere for both January and February are comparable both in magnitude and in structure to the respective ERA-40 anomaly indicates that the stratosphere of the model is subjected by a realistic extratropical tropospheric ENSO forcing, the matter of interest here. Further support to this conclusion is presented in Fig. 7, where the wENSO anomaly in the stationary eddies is shown. This result is expected because it has already been demonstrated that atmospheric general circulation models reproduce the extratropical tropospheric response to ENSO (Roeckner et al. 1996, and references therein; Strauss and Shukla 2002). The pattern of the wENSO temperature anomalies seen in the troposphere in Figs. 2 and 3 are also comparable to that deduced by satellite observations (Calvo Fernandez et al. 2004).
In the stratosphere, the magnitude of the simulated ensemble anomaly is somewhat smaller than that deduced from the ERA-40 data but, again, significant on broader areas. This is especially true in the upper stratosphere at $5 \mathrm{hPa}$.

The point of interest in the comparison of Figs. 2 and 3 is that the structure and the time evolution of the wENSO anomalies deduced from the ensemble mean of the simulations are consistent with those derived from the reanalysis. Figures 2 and 3 show a planetary wave response in the lower $(50 \mathrm{hPa})$ stratosphere in January, in agreement with Sassi et al. (2004). Here, in addition, we find that in the MAECHAM5 model as well as in the reanalysis there is a time-height change in the structure of the wENSO stratospheric response. While at $50 \mathrm{hPa}$ the January anomalies are characterized by the reported planetary wave one pattern, in February a polar warming (positive anomaly almost centered over the North Pole) takes over, suppressing the wave character of the anomalies poleward of $60^{\circ} \mathrm{N}$. In February at $50 \mathrm{hPa}$, the magnitude of the polar warming is also comparable in the ERA-40 and modelderived data. The fields at $5 \mathrm{hPa}$, although strongly modulated, suggest that the polar warming takes place higher up earlier in the winter. By looking at the October-April time series and throughout all of the middle atmosphere, this time-height structure is more obvious (not shown).

The time evolution from October to April of the polar warming and its effect on the zonal flow are illustrated in a compact way in Fig. 4 where the wENSO zonal-mean temperature anomalies at $80^{\circ} \mathrm{N}$ and zonal wind at $60^{\circ} \mathrm{N}$ are shown for the entire vertical domains of the model and up to $1 \mathrm{hPa}$ for the reanalysis. The largest anomalies in the ERA-40 data should be interpreted with caution (see section 3 ).

Concerning the model results, Fig. 4a reveals that the wENSO polar warming in the zonal-mean occurs first in the mesosphere (November), while the stratosphere is actually colder. A second and larger $(4 \mathrm{~K})$ polar warming descends from January to March, from the upper stratosphere to the lower stratosphere. This second temperature anomaly is statistically significant over a broad area. Above it there is a cooling, the manifestation of the dynamical response (involving gravity and planetary waves) of the mesosphere to any large-scale change in the stratosphere [see Manzini et al. (2003) for changes due to ozone depletion and Sassi et al. (2004) for changes due to ENSO, as in this case]. The pattern of the wENSO temperature anomaly in the reanalysis is remarkably similar to that deduced from the model results with the polar warming (albeit about twice the 

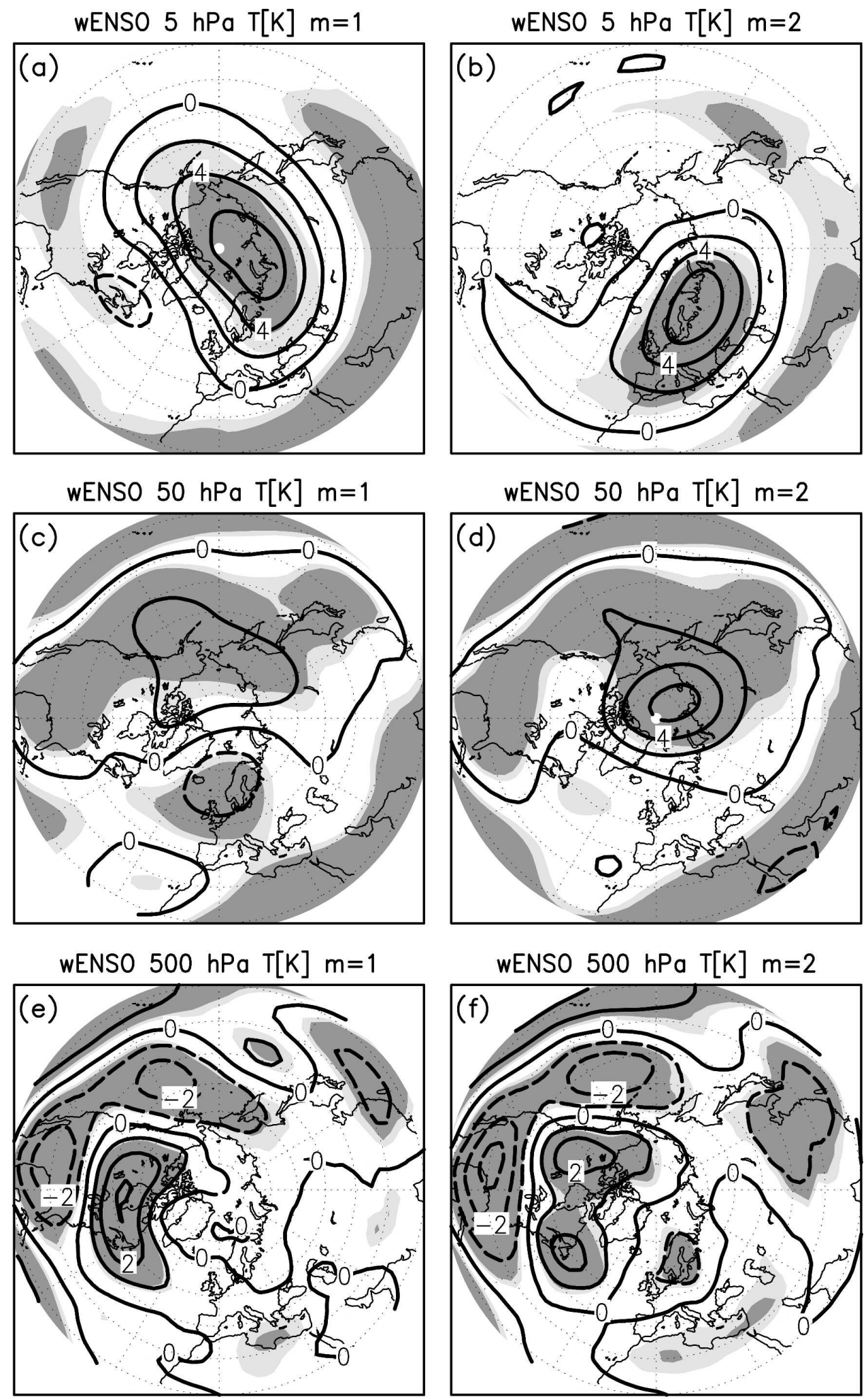

FIG. 2. (left) January and (right) February wENSO temperature anomaly from the ensemble of simulations (a), (b) at $5 \mathrm{hPa}$; (c), (d) at $50 \mathrm{hPa}$; and (e), (f) at $500 \mathrm{hPa}$. The contour at 5 and $50 \mathrm{hPa}$ is $2 \mathrm{~K}$ and at $500 \mathrm{hPa}$ it is $1 \mathrm{~K}$. Light and dark shades indicate statistical significance at the $95 \%$ and $99 \%$ levels, respectively. 
wENSO_ERA40 $5 \mathrm{hPa}$ T[K] $\mathrm{m}=1$
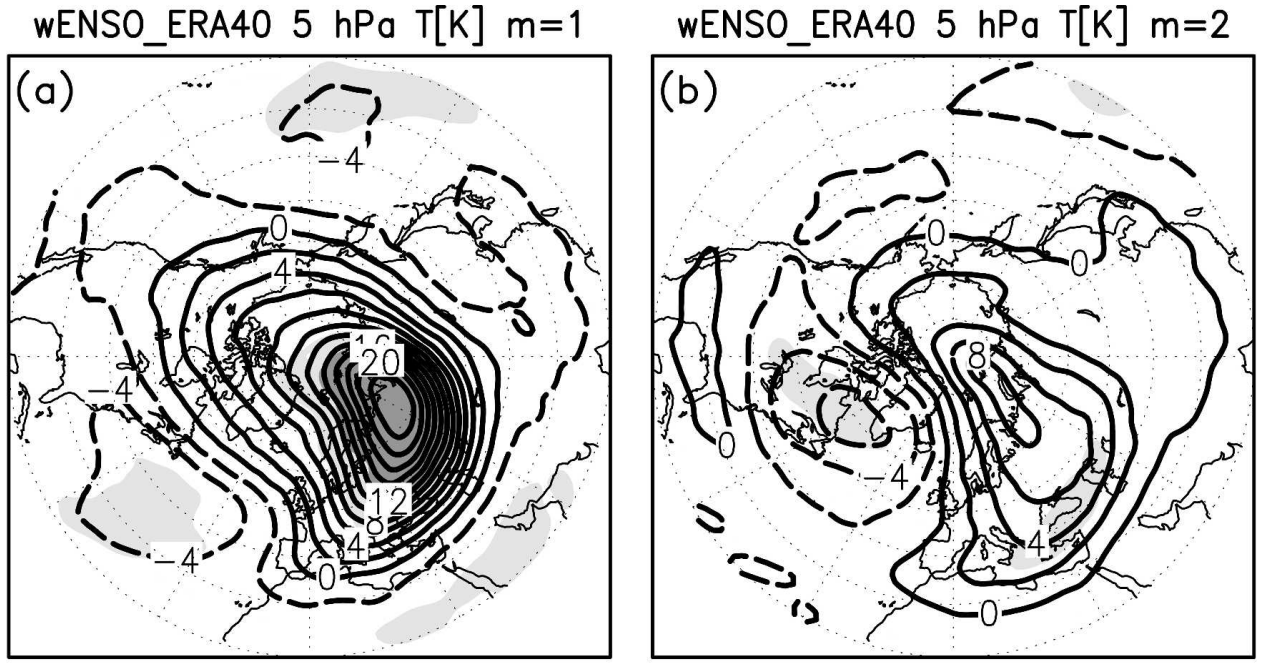

wENSO_ERA40 $50 \mathrm{hPa} T[\mathrm{~K}] \mathrm{m}=1$

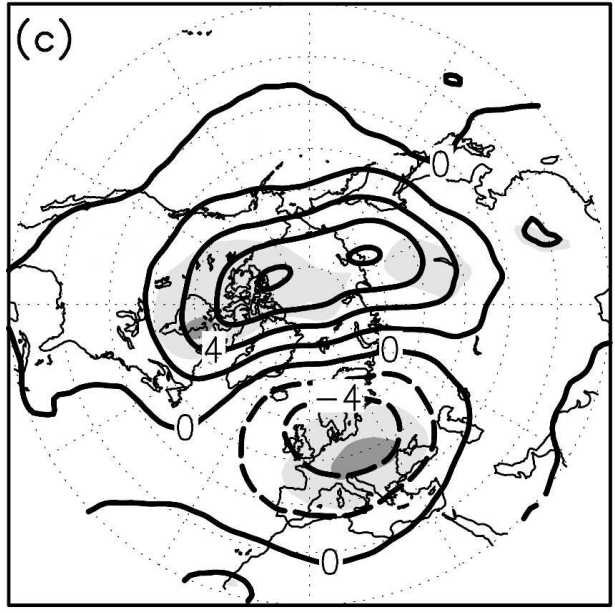

wENSO_ERA40 $50 \mathrm{hPa}$ T[K] $\mathrm{m}=2$

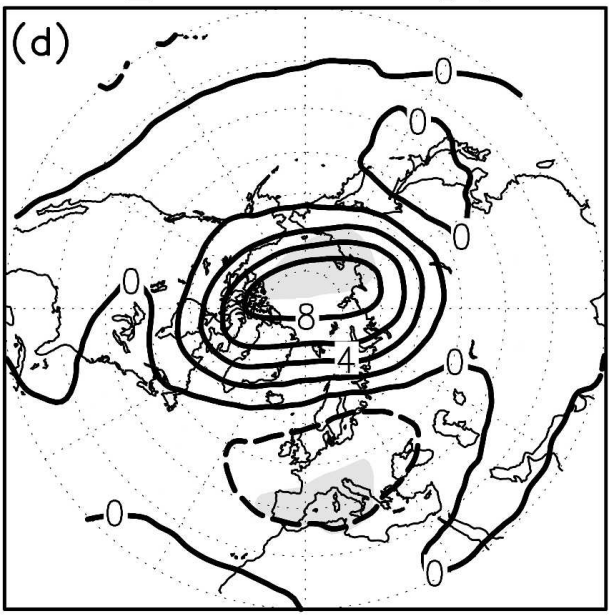

wENSO_ERA40 $500 \mathrm{hPa}$ T[K] $\mathrm{m}=1$

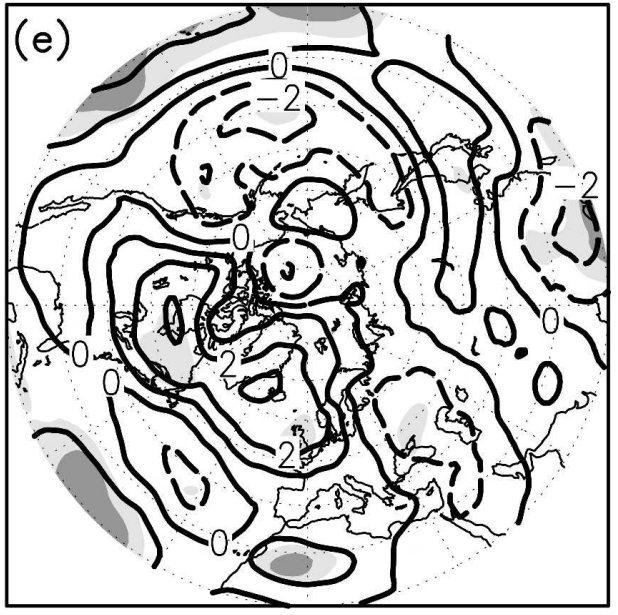

wENSO_ERA40 $500 \mathrm{hPa}$ T[K] $\mathrm{m}=2$

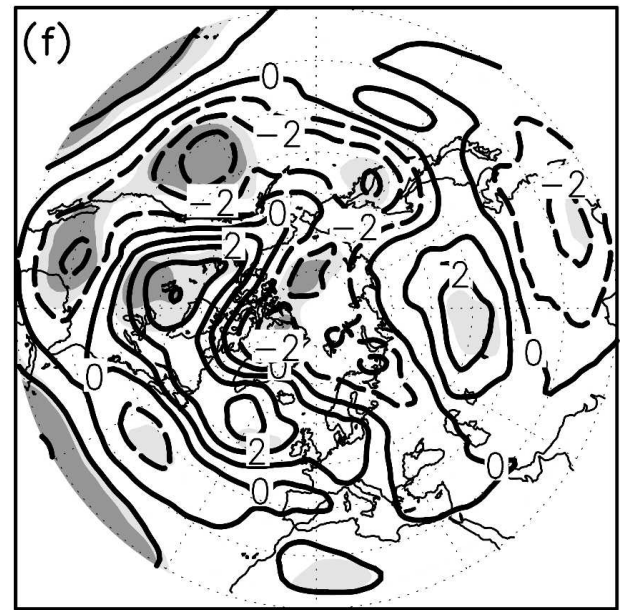

FIG. 3. Same as in Fig. 2, but from the ERA-40 data. 
(a) MAECHAM5 wENSO T[K] $80 \mathrm{~N}$

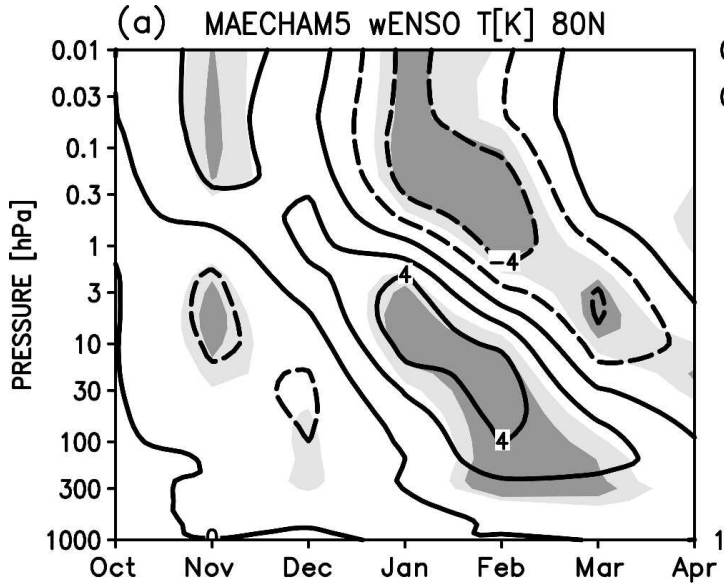

(c) MAECHAM5 wENSO U[m/s] $60 \mathrm{~N}$

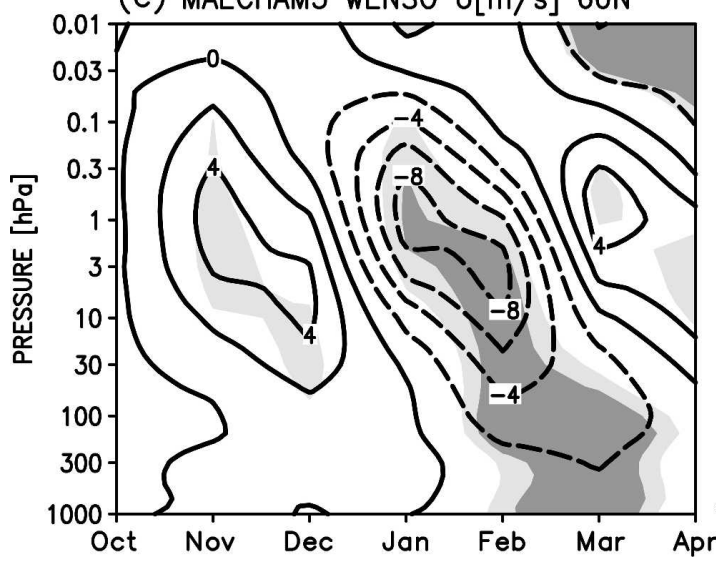

(b) ERA40 wENSO T[K] $80 \mathrm{~N}$

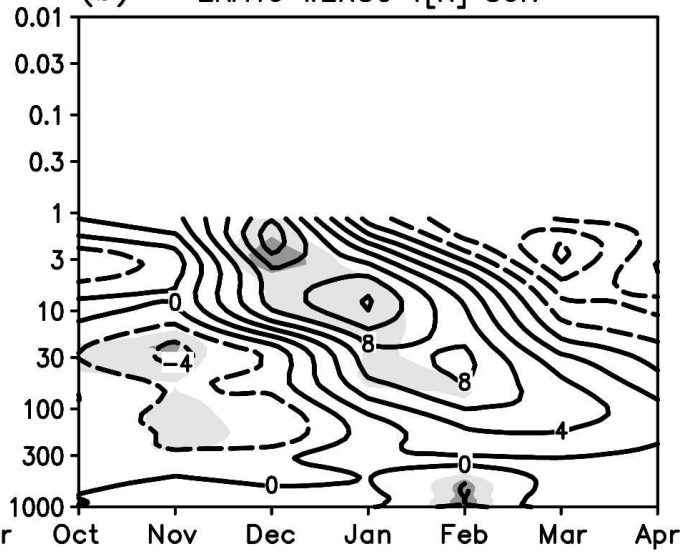

(d) ERA40 wENSO U[m/s] $60 \mathrm{~N}$

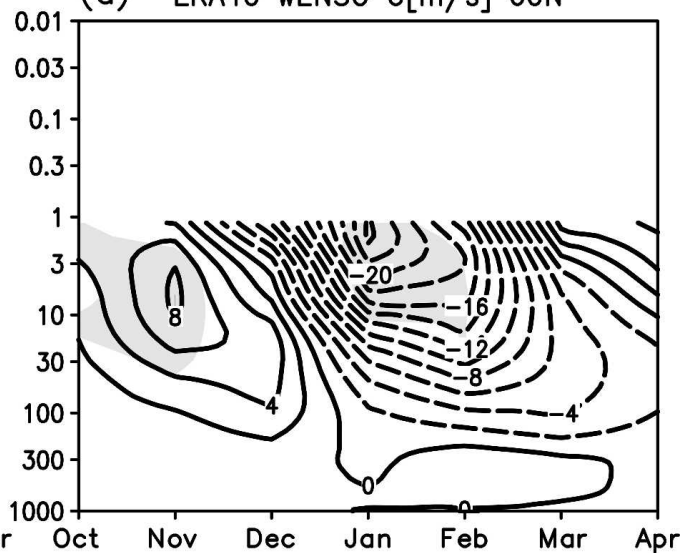

FIG. 4. October-April monthly zonal-mean wENSO anomaly. Temperature (contour: $2 \mathrm{~K}$ ) at $80^{\circ} \mathrm{N}$ for (a) the ensemble of simulations and (b) the ERA-40 data. Zonal wind (contour: $2 \mathrm{~m} \mathrm{~s}^{-1}$ ) at $60^{\circ} \mathrm{N}$ for (c) the ensemble of simulations and (d) the ERA-40 data. Light and dark shades indicate statistical significance at the $95 \%$ and $99 \%$ levels, respectively.

simulated one) descending from the uppermost levels of the domain, from December to March, and a cooling in the lower stratosphere in November and December. Unfortunately, there are no long-term reanalyses for a more confident comparison of the model results in the mesosphere.

That the reported polar warming is the manifestation of a change in the stratospheric circulation not confined to the immediate vicinity of the Pole is demonstrated by the time evolution of the wENSO anomalies in the zonal-mean zonal winds at $60^{\circ} \mathrm{N}$ (Fig. 4 , bottom) in both the model and the reanalysis data. Again, the patterns of the wENSO anomaly for the two datasets are remarkably similar. Consistent with the polar anomalies, in the stratosphere the zonal-mean zonal wind anomalies at $60^{\circ} \mathrm{N}$ are characterized by a strengthening of the polar vortex in November and December and a weakening in February and March.

In the lower stratosphere, the stratospheric response to the warm ENSO phenomenon can also be interpreted as a shift in the seasonal cycle (coldest month December instead of January). In addition, the model results indicate that in late winter/early spring the stratospheric response to the warm ENSO phenomenon can influence the status of the tropopause and even the troposphere, inducing small but significant changes down to $300 \mathrm{hPa}$ in the temperature (from 2 to $4 \mathrm{~K}$ ) and to the surface in the zonal winds.

Taking into consideration the reported shortcomings of the reanalysis, it is of interest that both the model and the reanalysis present a coherent picture of the stratospheric changes associated with the occurrence of the warm ENSO phenomenon, both from synoptic maps and from the seasonal evolution of the zonalmean flow. Namely, the behavior of the reanalyzed and modeled zonal wind fields are a clear manifestation of an enhancement of the downward propagation of wave-mean-flow interaction in the middle stratosphere, 
given an enhancement of the tropospheric forcing of upward propagating wave activity. From the results presented here a distinct response in the zonal-mean flow emerges, not shown previously.

The cENSO temperature anomalies at 500, 50, and 5 hPa are depicted in Fig. 5, for February only. The comparison of the model and reanalysis data at $500 \mathrm{hPa}$ shows that the model reproduces the structure and magnitude of the temperature anomaly in the troposphere, positive anomalies over the northern Pacific Ocean and negative anomalies in the polar regions, north of the positive anomalies. However, in comparison to the wENSO temperature anomalies in the troposphere, the cENSO anomalies appear to be smaller (of about a factor 2) over the North American continent (although this difference is clearer in the model data, it is also discernible in the reanalysis). From Fig. 5 it is deduced that the response in the stratosphere to cold ENSO events is either negligible or not distinguishable from the internal variability of the polar vortex. This result is found for both the model and the reanalysis and supports the fact that Sassi et al. (2004) had difficulty finding a signal for the cold ENSO events in their statistical analysis. Consistently, the cENSO anomalies (shown only for the model, Fig. 6) in the zonal-mean temperature at $80^{\circ} \mathrm{N}$ and zonal wind at $60^{\circ} \mathrm{N}$ are relatively weak, confined to the stratosphere, and do not propagate downward.

\section{Stationary eddies}

In the discussion of Fig. 5 it is mentioned that the extratropical tropospheric anomaly during cold ENSO events appears to be smaller in magnitude over the North American continent. This result is consistent with the smaller SST anomaly in the tropical Pacific for the cold events, a typical characteristic of the ENSO cycle. To investigate further the connection between the troposphere and the stratosphere during the ENSO cycle, the wENSO and cENSO anomalies in the stationary eddy heights are compared, within each other and between the model and reanalysis, in Figs. 7 and 8 .

The typical tropospheric response $(500 \mathrm{hPa}$, Fig. 7$)$ in the December-February (DJF) stationary eddy height is found in the model, for both the wENSO and cENSO anomalies. This result is supported by the comparison with the ERA-40 data shown also in Fig. 7 and also by comparison with published and comprehensive analysis of the influence of ENSO on the extratropics from NCEP reanalysis (Hoerling et al. 1997).

Namely, in the case of the warm ENSO composite, it is noted that over the North Pacific Ocean the wENSO anomaly indicates an eastward elongation of the northwest Pacific trough. The magnitude of this negative anomaly is virtually identical in the model and in the ERA-40 data. Eastward of it, the positive anomaly over the North American continent has a smoother and larger-scale character for the ensemble results, possibly more representative of the ENSO signal. This latter positive anomaly therefore results in a weakening of the North American-western Atlantic trough seen in the full field (Fig. 7, bottom).

Given that the wENSO change in the structure (and magnitude) of the eddies is persistent in time (e.g., seasonal; it is seen in winter mean), it may enhance the tropospheric forcing of stationary planetary wave one, which in turn may propagate upward into the stratosphere. This behavior is, indeed, demonstrated in Fig. 8, a longitude-pressure cross section of the stationary height eddies and their respective anomalies at $50^{\circ} \mathrm{N}$. Figure 8 (top panels) shows that the wENSO positive tropospheric anomaly at $60^{\circ} \mathrm{W}$ virtually compensate for the negative eddy height (at $60^{\circ} \mathrm{W}$, bottom panel) for both model and reanalysis. Thereafter, in the stratosphere the vertical structure of the wENSO eddy height anomaly, deduced from the ensemble of simulations, clearly shows an enhancement of stationary wave one and also of its westward tilt (cf. Figs $8 \mathrm{a}$ and 8e). These model results are consistent with the early observational evidence suggesting an enhancement of the Aleutian high in the lower stratosphere for warm ENSO events (van Loon and Labitzke 1987; Hamilton 1993a) and with the response to warm ENSO events deduced from the ERA-40 data and shown in Figs. 7 and 8. However, the planetary wave enhancement in the ERA-40 data does not appear to be confined to wave one. This result is found also for the NCEP/CPC data. Given that the ensemble mean smooths the character of the fields, the model results suggest that the essential response of the stratosphere to the warm ENSO phenomenon is a tropospheric-driven planetary wave one.

In the troposphere (Fig. 7, middle), the modeled cENSO eddy height anomaly pattern is almost in quadrature with the respective wENSO anomaly and its amplitude about a factor of 2 less. Note that the model results actually compare better than the ERA-40 results with Hoerling et al. (1997), presumably because a relatively small number of cases of cold events have been included in the ERA-40 composite. The structure of the cENSO eddy height anomaly (opposite in sign to the climatological stationary eddies between $120^{\circ} \mathrm{E}$ and $60^{\circ} \mathrm{W}$ ) therefore indicates a general weakening of the tropospheric forcing contributing to the stratospheric planetary wave one (cf. middle and bottom panels, Fig. 8). Therefore, the negligible response in the stratosphere to cold ENSO events can be explained by a 

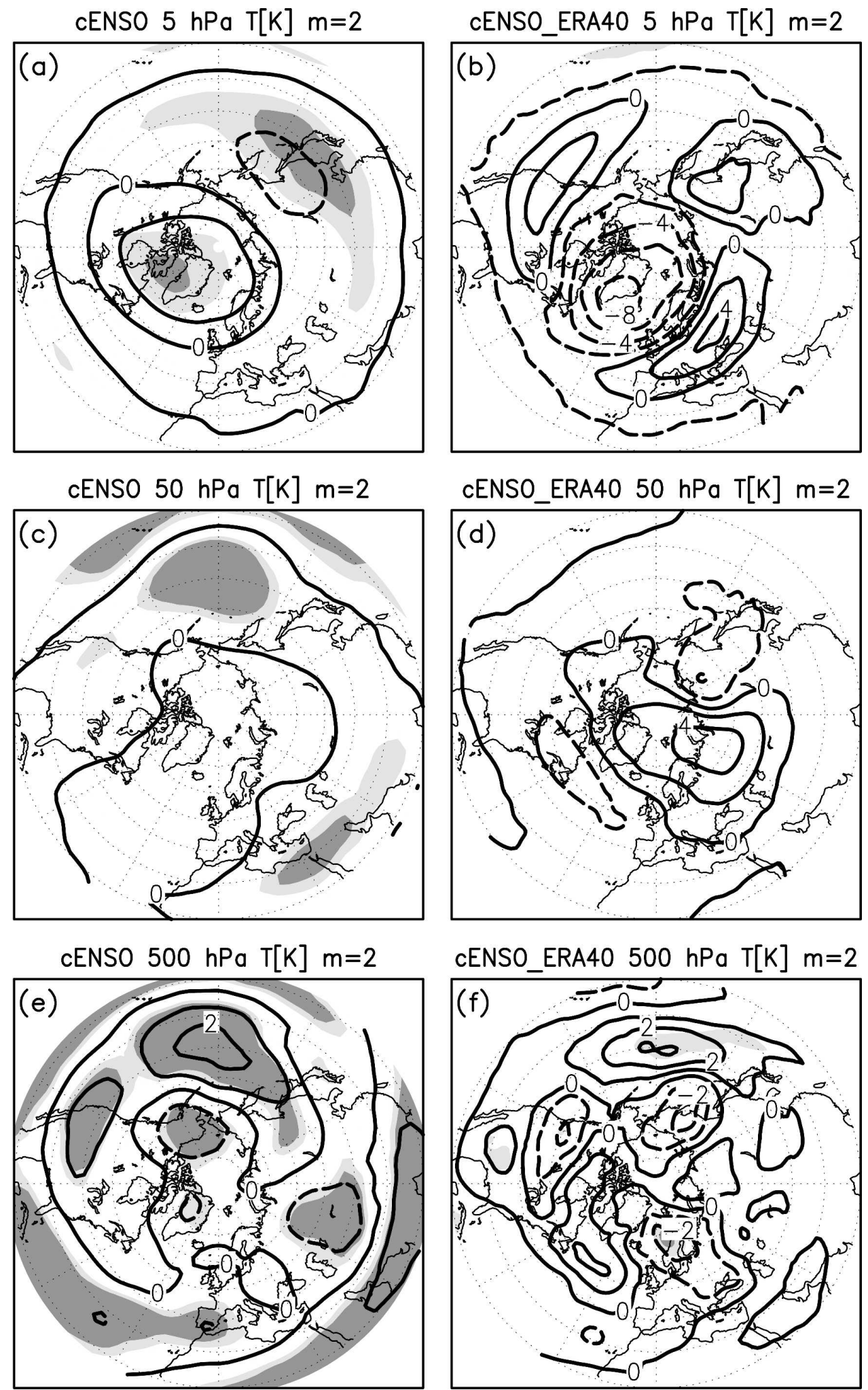

FIG. 5. February cENSO temperature anomaly at $5 \mathrm{hPa}$ for (a) the ensemble of simulations and (b) the ERA-40 data, at $50 \mathrm{hPa}$ for (c) the ensemble of simulations and (d) the ERA-40 data, and at $500 \mathrm{hPa}$ for (e) the ensemble of simulations and (f) the ERA-40 data. The contour at 5 and $50 \mathrm{hPa}$ is $2 \mathrm{~K}$ and at $500 \mathrm{hPa}$ it is $1 \mathrm{~K}$. Light and dark shades indicate statistical significance at the $95 \%$ and $99 \%$ levels, respectively. 

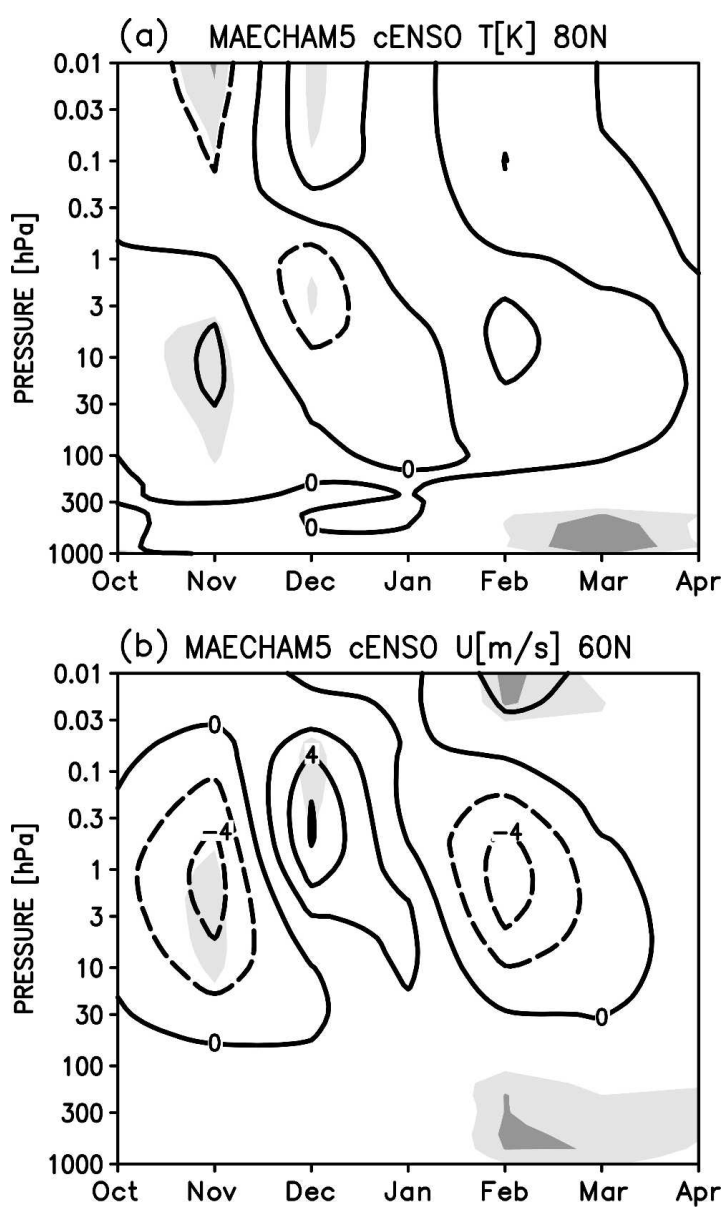

FIG. 6. October to April monthly zonal-mean cENSO anomaly: (a) temperatures (contour: $2 \mathrm{~K}$ ) at $80^{\circ} \mathrm{N}$ and (b) zonal winds (contour: $2 \mathrm{~m} \mathrm{~s}^{-1}$ ) at $60^{\circ} \mathrm{N}$. Results from the ensemble of simulations. Light and dark shades indicate statistical significance at the $95 \%$ and $99 \%$ levels, respectively.

combination of both weaker and less large-scale forcing than that determining the climatological average.

\section{SST influence on systematic temperature changes}

Heterogeneous chemistry of ozone depletion is sensitive to the ambient temperature (Solomon 1999). It is therefore of great concern to determine the reasons for the recent systematic temperature changes observed in the lower stratosphere in the Arctic in winter and spring (Ramaswamy et al. 2001). In particular, it is of interest to determine if the polar cooling can arise from dynamical processes only or if an interaction between dynamics and chemistry is necessary, possibly inclusive of perturbed atmospheric composition (Manzini et al. 2003, and references therein).

To address the possibility that recent systematic changes in the polar temperature arise from SST varia- tions, the seasonal evolution of the monthly zonalmean temperature difference between the two decades considered is shown, in Fig. 9, at $80^{\circ}$ N. Figure 9 shows a cooling in the stratosphere and a warming in the mesosphere in December, January, March, and April. The opposite sign of the change in the stratosphere and mesosphere is the typical manifestation of a temperature difference of dynamical origin, forced from below. The positive difference in the stratosphere in February can be related to the particularly large ENSO event of 1998 (also clearly visible in Fig. 10). It is interesting to note that the stratospheric cooling extends below $30 \mathrm{hPa}$ only in January when the warm ENSO influence is negligible (Fig. 4). The temperature difference in the winter average temperature at $50 \mathrm{hPa}$ for the latitudinal band $60^{\circ}-90^{\circ} \mathrm{N}$ is, however, somewhat small, between -0.1 and $-0.2 \mathrm{~K}$ (not shown). Observations indeed indicate a change about an order of magnitude larger (Ramaswamy et al. 2001).

To further illustrate the character of the ensemblemean time series obtained from the MAECHAM5 model, the monthly zonal-mean temperature anomaly at $50 \mathrm{hPa}$ is shown in Fig. 10, averaged over two latitudinal bands (northern polar and tropical, respectively). The linear trend computed for the whole time record of the simulation ensemble mean is virtually null, indicating that on long time scales the dynamical changes induced by the SSTs do not produce a systematic change. On shorter time scales, it is possible to identify small trends, mainly the results of internal variations at interannual scales and the influence of ENSO, reported in the previous section. The dynamical origin of the temperature anomalies seen in Fig. 10 is demonstrated by the opposite sign in the anomalies in the polar and tropical latitudinal bands (Salby and Callaghan 2003).

\section{Summary and conclusions}

The newly configured middle atmosphere MAECHAM5 general circulation model has been presented and applied to the problem of evaluating the Northern Hemisphere polar winter stratospheric response to SST interannual variations. Three aspects of influence have been addressed, namely, the SST influence on the climatological mean of the zonal-mean zonal wind, the influence of ENSO variations, and the influence on systematic changes (trends) in temperature. All of the results presented are based on monthly means and are restricted to the boreal extended winter season. The main results obtained are as follows.

1) It is not straightforward to deduce an influence of the SSTs on the time average state in the polar re- 


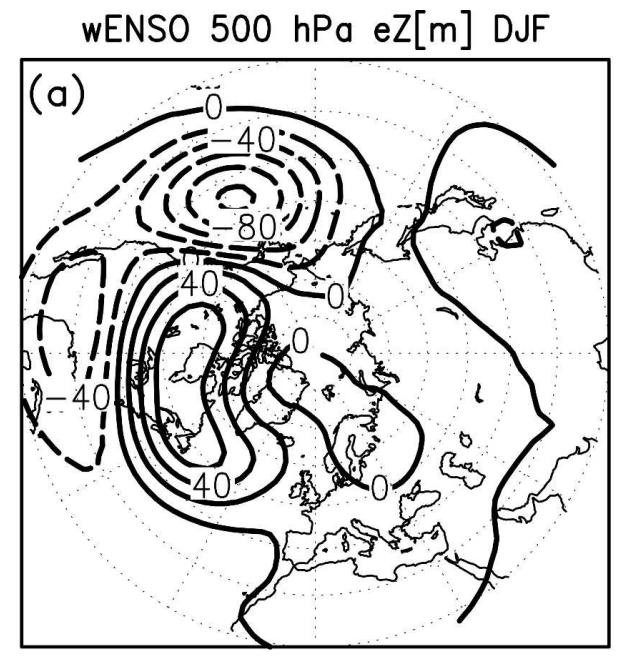

wENSO_ERA40 $500 \mathrm{hPa}$ eZ[m] DJF

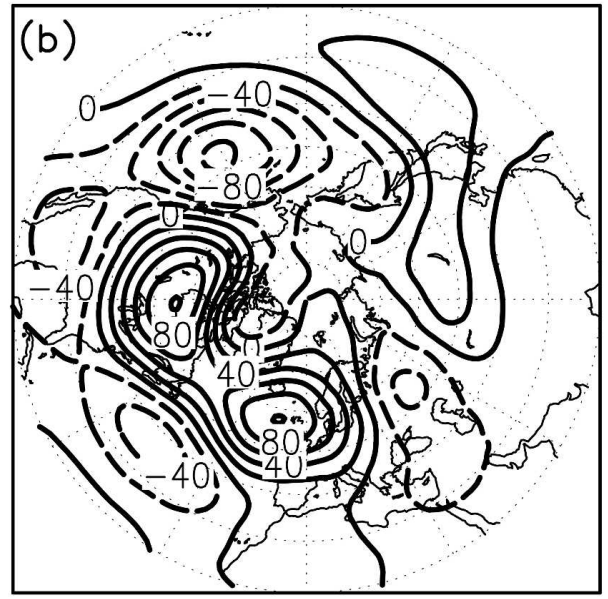

cENSO $500 \mathrm{hPa}$ eZ[m] DJF

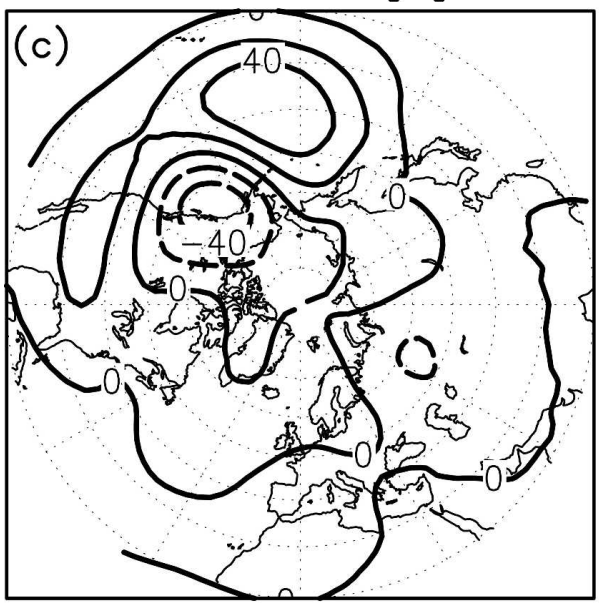

cENSO_ERA40 $500 \mathrm{hPa}$ eZ[m] DJF

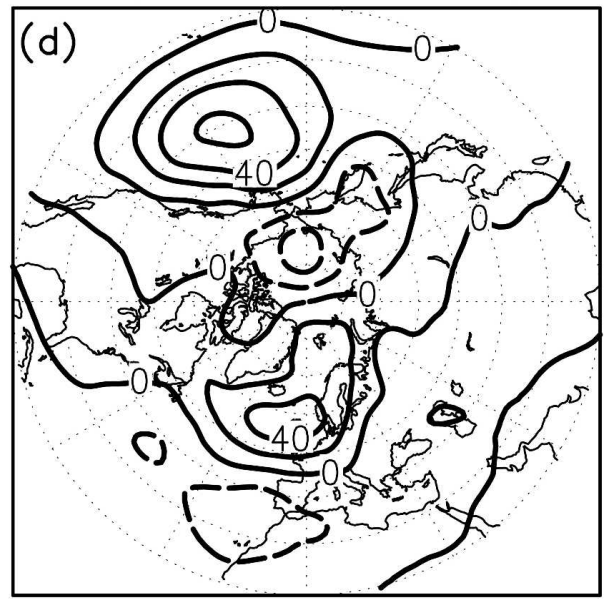

NEUTRAL $500 \mathrm{hPa}$ eZ[m] DJF

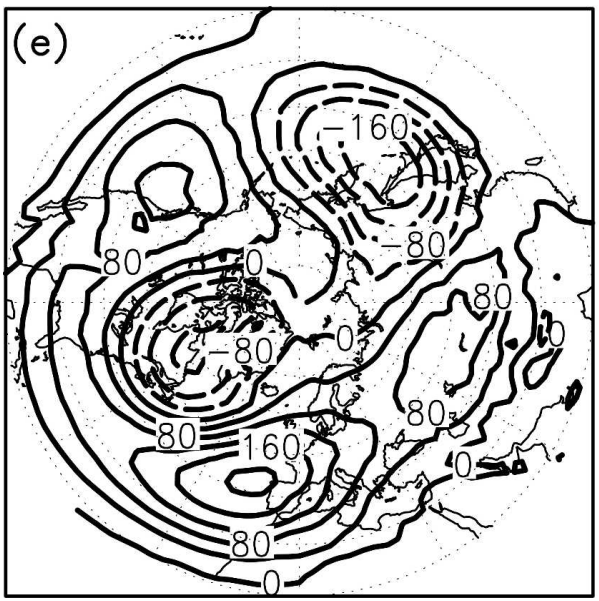

NEUTRAL_ERA40 $500 \mathrm{hPa}$ eZ[m] DJF

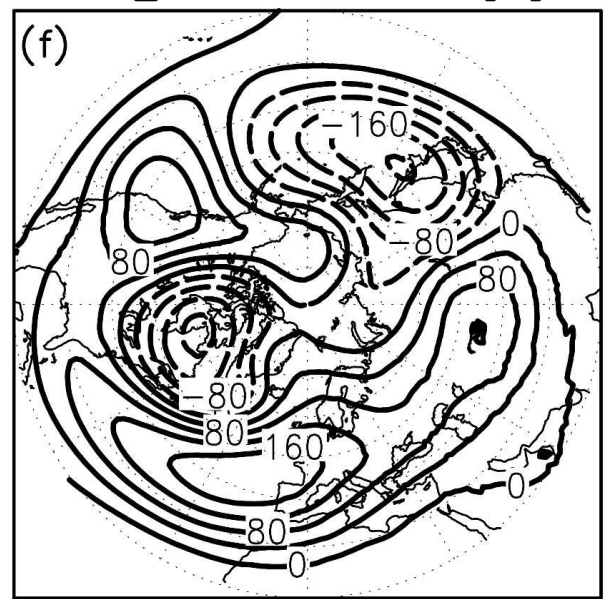

FIG. 7. DJF stationary eddy height anomaly at $500 \mathrm{hPa}$ wENSO anomaly from (a) the ensemble of simulations and (b) the ERA-40 data; cENSO anomaly from (c) the ensemble of simulations and (d) the ERA-40 data (contour: $20 \mathrm{~m}$ ). DJF stationary eddy height for the NEUTRAL composite from (e) the ensemble of simulations and (f) the ERA-40 data (contour: $40 \mathrm{~m}$ ). 

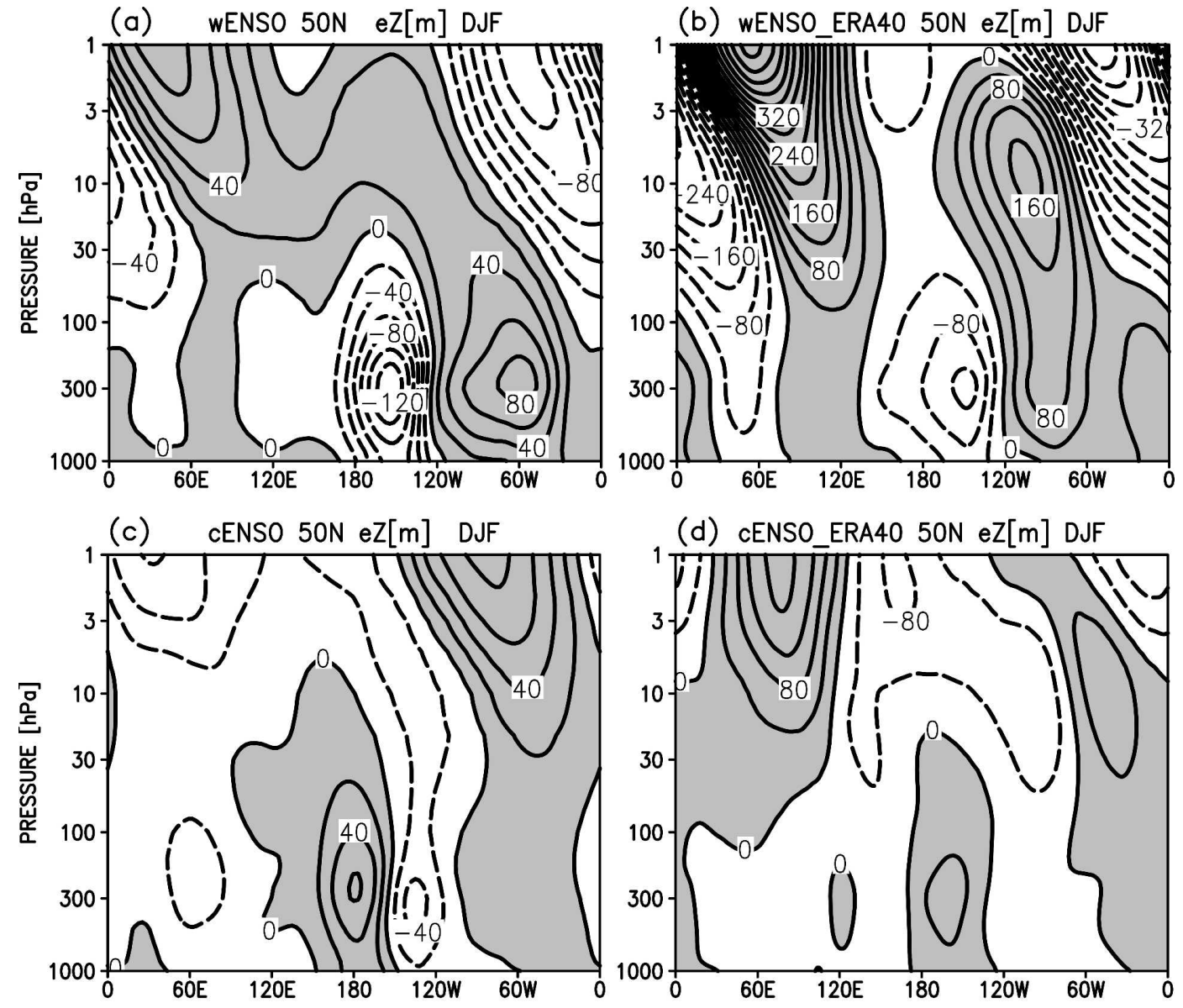

(d) cENSO_ERA40 50N eZ[m] DJF
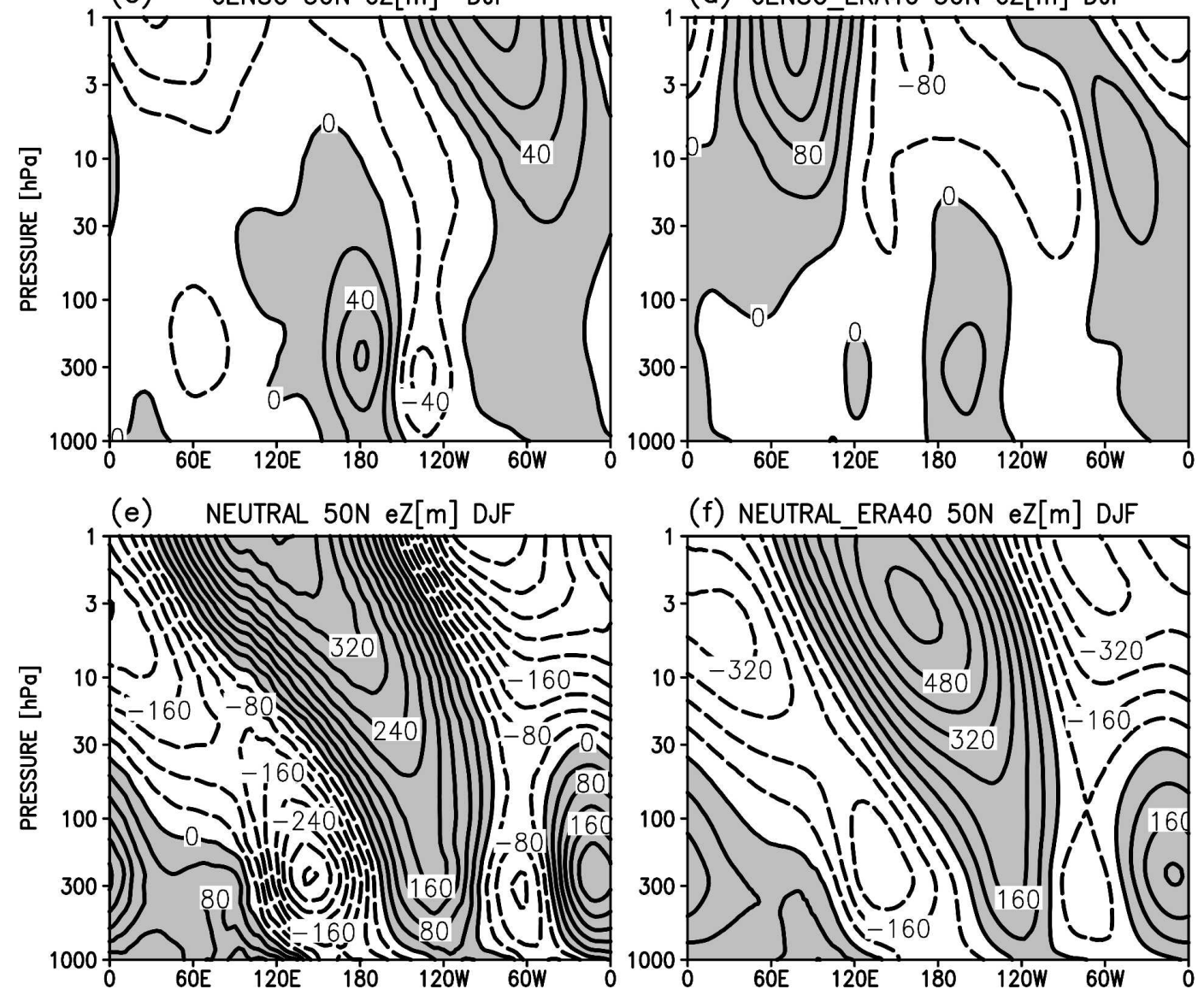

(f) NEUTRAL_ERA40 50N eZ[m] DJF

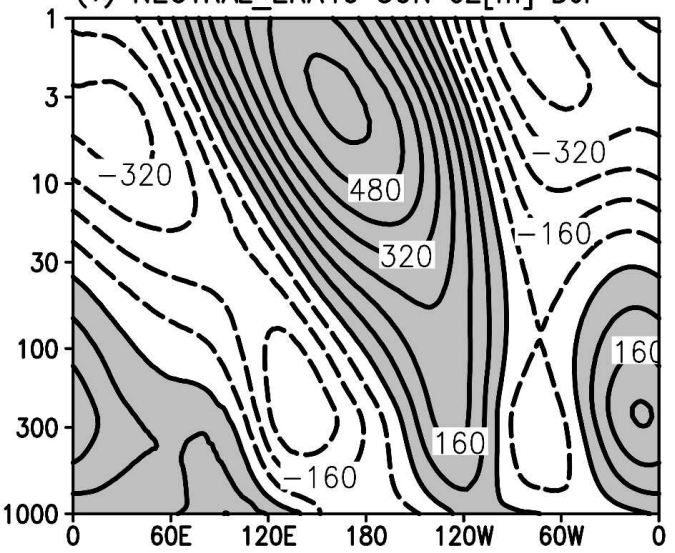

FIG. 8. DJF stationary eddy height anomaly at $50^{\circ} \mathrm{N}$ : wENSO anomaly from (a) the ensemble of simulations and (b) the ERA-40 data; cENSO anomaly from (c) the ensemble of simulations and (d) the ERA-40 data (contours: $20 \mathrm{~m}$ at left and $40 \mathrm{~m}$ at right). DJF stationary eddy height for the NEUTRAL composite from (e) the ensemble of simulations and (f) the ERA-40 data (contour: $40 \mathrm{~m}$ at left and $80 \mathrm{~m}$ at right). Shades indicate positive values.

gions because of the large internal variability of the polar stratosphere, a realistic feature. These results appear to be in contrast with Braesicke and Pyle (2004) in the sense that statistically significant dif- ferences are not found from the MAECHAM5 results. However, qualitatively the MAECHAM5 results for the stratospheric polar night jet are consistent with Braesicke and Pyle (2004). The ensemble 


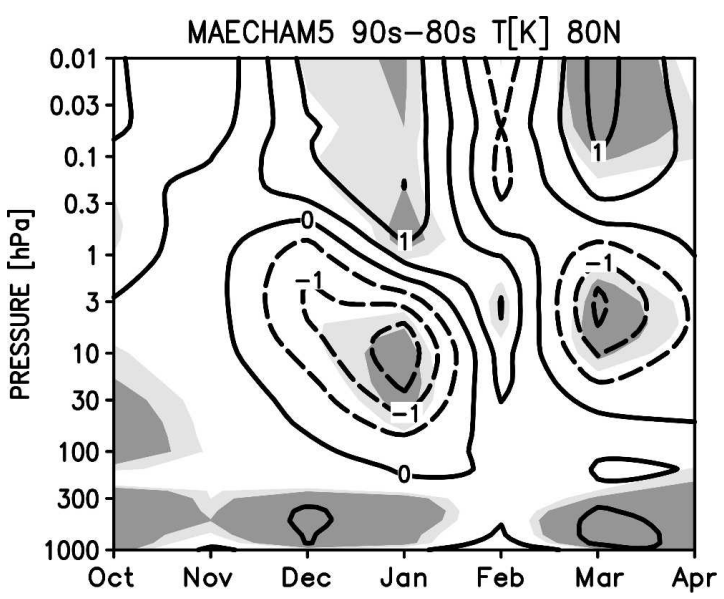

FIG. 9. October-April monthly zonal-mean temperature difference between the climatological means of the first and the second decade (1990s minus 1980s) from the ensemble of simulations at $80^{\circ} \mathrm{N}$ (contour: $0.5 \mathrm{~K}$ ).

of simulations from the MAECHAM5 model suggest an influence on the climatological flow in the subtropical and tropical stratosphere.

2) The distinct emergence of a stratospheric response to warm ENSO events, is explained by an enhancement of the tropospheric driving of a stationary planetary wave one, associated with known structural changes in the stationary eddies in the western Pacific and North America regions during ENSO events (Hoerling et al. 1997). These results concerning the stratospheric response are in agreement with earlier observational evidence (van Loon and Labitzke 1987; Hamilton 1993a), which associated the stratospheric response to a warm ENSO and to a quasi-stationary amplification of the Aleutian high, as well as with the modeling work of Hamilton (1993b) and Sassi et al. (2004). The novel result presented here is the characterization of the seasonal evolution of the stratospheric response and its manifestation on the zonal-mean temperature and zonal wind, demonstrating a large-scale effect of warm ENSO events. These results are supported not only by the analysis of the simulation ensemble but also by the ERA-40 diagnostics. Namely, in the lower stratosphere the planetary wave enhancement takes place in early winter and its effect on the zonal mean progresses downward in time, from the mesosphere to the upper troposphere, in agreement with wavemean flow interaction theory (Andrews et al. 1987). Moreover, both the MAECHAM5 model results and the reanalysis data show that in early winter, during the wave enhancement phase in the stratosphere, the zonal-mean stratospheric flow is characterized by a weak cooling and a weak strengthening of the zonal wind in the lower stratosphere. It is only in February and March that the lower Arctic stratosphere is characterized by a relatively large warming (4 $\mathrm{K}$ on average) and weakening of the zonal wind. Overall, our results are consistent with Sassi et al. (2004) in spring, while they might not be in early winter and concerning the downward propagation of the zonal-mean anomalies. Given that the driving aspect (the planetary wave enhancement) of the response to the warm ENSO and its extension to the stratosphere is captured by both MAECHAM5 and the Whole-Atmosphere Community Climate Model

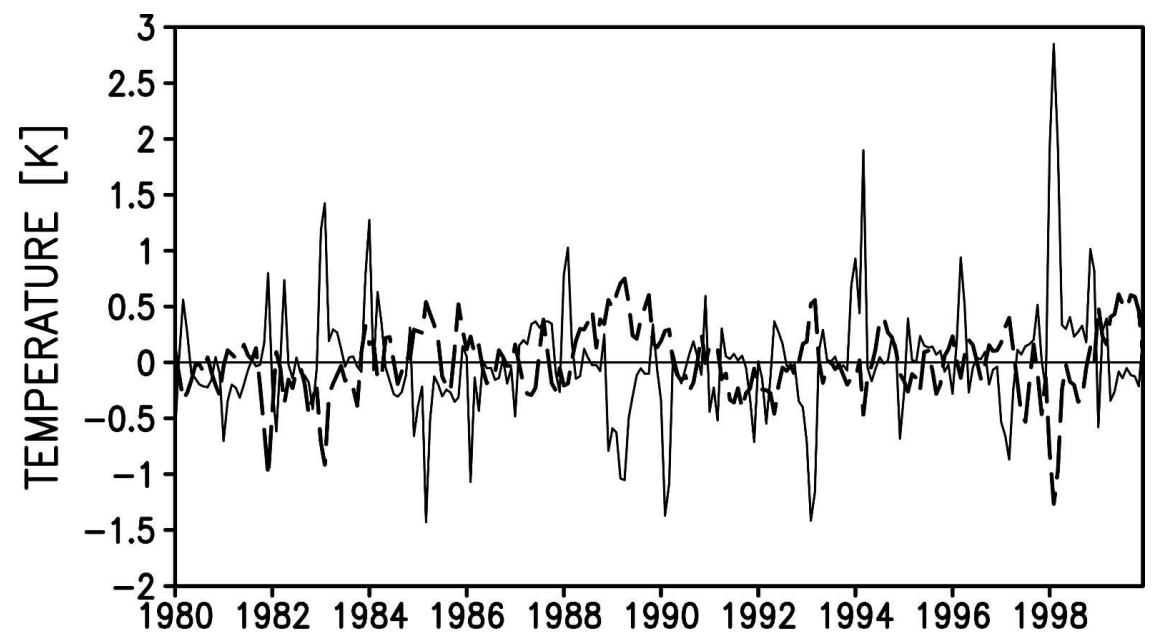

FIG. 10. Monthly zonal-mean temperature anomaly from the climatological mean of the ensemble of simulations at $50 \mathrm{hPa}$, averaged over $50^{\circ}-90^{\circ} \mathrm{N}$ (solid) and $30^{\circ} \mathrm{S}-50^{\circ} \mathrm{N}$ (dashed). 
(WACCM: the GCM used by Sassi et al.), it is plausible that such differences may be at least in part due to the respective differences in experimental design and, consequently, in the selection of ENSO events and their analysis.

3) Negligible stratospheric response to cold ENSO events is explained by a weak reduction of the climatological tropospheric stationary waves. This result is consistent with a weaker forcing (the SST anomalies for the cold ENSO events considered are on average somewhat smaller than that for the warm ENSOs) and with the pattern of the extratropical tropospheric response to cold ENSO events previously reported (Hoerling et al. 1997). Consequently, the influence induced by cold ENSO events does not appear to be distinguishable from internal variability. From these results, average cold ENSO events cannot precondition the lower stratosphere to cold conditions favorable to ozone depletion in an atmosphere perturbed by ozone-depleting substances.

4) Concerning systemic changes in the lower stratosphere induced by SST variations alone, it appears that a cooling (albeit weak) can be deduced from the ensemble of simulations only in January when the warm ENSO influence is minimal. This result points to the importance of developing models able to simulate the atmospheric circulation and its variability in a comprehensive way. Indeed, although the current results can appear in contrast to Lahoz (2000), the fact that we recover a cooling only in January (when ENSO influence is minimal) can be thought to be consistent with the fact that the simulations presented in Lahoz did produce a large trend but did not support an ENSO influence. If these differences are genuine, they would indicate that, so far, the sensitivity of models to external forcing is not comparable.

Another interesting new aspect revealed in this work is the evidence for a downward feedback in spring — via zonal-mean anomalies - of the stratospheric response to warm ENSO events on the extratropical troposphere, manifested as a statistical significant difference in February and March in the zonal-mean wind down to the surface. This downward feedback appears to require a sustained forcing during the winter, given that comparable stratospheric perturbation in November for both warm ENSO and cold ENSO events do not appear to propagate downward for the cold ENSO anomalies. To detail these aspects is outside the scope of this work and can be addressed in future works.

In addition, further analysis of the ensemble of simulations performed with MAECHAM5 can address simi- lar questions for the Southern Hemisphere. In this case, consideration of simulations that include prognostic ozone would be of interest, given the known role of ozone variations in the Antarctic lower stratosphere (Steil et al. 2003; Manzini et al. 2003, and references therein).

It may also be of interest to perform the reported analysis on an ensemble of simulations covering a longer time period to include a larger number of warm and cold ENSO cases and assess the generality of the results obtained. From this work, confidence in the results is based on the fact that the simulated midlatitude tropospheric response to both warm and cold ENSOs is well captured by the relatively few cases considered, for instance in comparison with the observed response reported by Hoerling et al. (1997) that is based on a larger number of events.

A question not addressed here is also the influence of the SST on the North Atlantic Oscillation/Artic Oscillation and their troposphere-stratosphere connection. This last issue may require an analysis of the character of the internal variability and how it relates to forced variability (Quadrelli and Wallace 2002). Although interesting, these issues may require ensemble simulations covering a much longer period (Hurrell et al. 2004) than that considered here and are therefore beyond the scope of this work.

In summary, concerning ENSO influence, this work supports the independent results of Hamilton (1993b) and Sassi et al. (2004). In particular, this work and Sassi et al. are broadly consistent with each other and with observations. However, the limited analysis based on the Unified Model (UM) (Braesicke and Pyle 2004; Lahoz 2000) apparently suggests a much smaller influence of SST variations associated with ENSO. In addition to different model response and experimental design, a possibility is that the SST and SIC used are not comparable with each other. Further differences between MAECHAM5 and the UM concern systematic changes induced by dynamical processes, with MAECHAM5 apparently much less sensitive. More stringent model intercomparisons are necessary to solve these issues. In particular, simulations with identical forcing are called for.

Concerning the ongoing discussion on temperature trends in the lower stratosphere during northern winter, it is noted that in this work (as in Lahoz) a constant specified ozone was used. Given the influence on the ozone distribution of dynamical changes [as highlighted by Salby and Callaghan (2003)], an open question may be if the inclusion of prognostic ozone in a model may lead to a larger response in the polar lower stratosphere. The question of whether an ozone feedback 
may amplify the dynamical variations may be relevant both for the case of unperturbed (pre-ozone hole) and perturbed (high chlorine) atmospheric composition and can be addressed in the future with the so-called chemistry-climate models.

Acknowledgments. The ECHAM5 modeling group is acknowledged for invaluable technical support. We are grateful to Antonio Navarra for reading the manuscript and suggesting improvements. Deutsches Klimarechenzentrum (DKRZ) and ECMWF, Special Project: Middle Atmosphere Modeling, are acknowledged for providing computing power.

\section{REFERENCES}

Andrews, D. G., J. R. Holton, and C. B. Leovy, 1987: Middle Atmospheric Dynamics. Academic Press, 489 pp.

Baldwin, M. P., and D. O'Sullivan, 1995: Stratospheric effects of ENSO related tropospheric circulation anomalies. J. Climate, 8, 649-667.

Boville, B. A., 1995: Middle atmosphere verson of the CCM2 (MACCM2): Auunual cycle and interannual variability. $J$. Geophys. Res., 100, 9017-9039.

Braesicke, P., and J. A. Pyle, 2004: Sensitivity of dynamics and ozone to different representations of SSTs in the Unified Model. Quart. J. Roy. Meteor. Soc., 99, doi:10.1256/qj.03.999.

Calvo Fernandez, N., R. R. Garcia, R. Garcia Herrera, D. Gallego Puyol, L. Gimeno Presa, E. Hernandez Martin, and P. Ribera Rodriguez, 2004: Analysis of the ENSO signal in tropospheric and stratospheric temperatures observed by MSU, 1979-2000. J. Climate, 17, 3934-3946.

Charron, M., and E. Manzini, 2002: Gravity waves from fronts: Parameterization and middle atmosphere response in a general circulation model. J. Atmos. Sci., 59, 923-941.

Fortuin, J. P. F., and H. Kelder, 1998: An ozone climatology based on ozondesonde and satellite measurements. J. Geophys. Res., 103, 31 709-31 734.

Giorgetta, M.A., E. Manzini, and E. Roeckner, 2002: Forcing of the quasi-biennial oscillation from a broad spectrum of atmospheric waves. Geophys. Res. Lett., 29, 1245, doi:10.1029/ 2002GL014756.

— - — - M. Esch, and L. Begtsson, 2006: Climatology and forcing of the quasi-biennial oscillation in the MAECHAM5 model. J. Climate, 19, 3882-3901.

Gray, L. J., 2003: The influence of the equatorial upper stratosphere on stratospheric sudden warmings. Geophys. Res. Lett., 30, 1166, doi:10.1029/2002GL016430.

Hamilton, K., 1993a: An examination of observed Southern Oscillation effects in the Northern Hemisphere stratosphere. $J$. Atmos. Sci., 50, 3468-3473.

_ 1993b: A general circulation model simulation of El Niño effects in the extratropical Northern Hemisphere stratosphere. Geophys. Res. Lett., 20, 1803-1806.

—, R. J. Wilson, J. D. Mahlman, and L. J. Umscheid, 1995: Climatology of the SKYHI troposphere-stratosphere-mesosphere general circulation model. J. Atmos. Sci., 52, 5-43.

Hoerling, M. P., A. Kumar, and M. Zhong, 1997: El Niño, La Niña, and the nonlinearity of their teleconnections. J. Climate, 10, 1769-1786.
Holton, J. R., and C. Mass, 1976: Stratospheric vacillation cycles. J. Atmos. Sci., 33, 2218-2225.

Hurrell, J. W., M. P. Hoerling, A. S. Phillips, and T. Xu, 2004: Twentieth century North Atlantic climate change. Part I: Assessing determinism. Climate Dyn., 23, doi:10.1007/s00382004-0432-y.

Kodera, K., and Y. Kuroda, 2000: A mechanistic model study of slowly propagating coupled stratosphere-troposphere variability. J. Geophys. Res., 105, 12 361-12 370.

_, M. Chiba, H. Koide, A. Kitoh, and Y. Nikaidou, 1996: Interannual variability of the winter stratosphere and troposphere in the Northern Hemisphere. J. Meteor. Soc. Japan, 74, 365-382.

Labitzke, K., 1981: Stratospheric-mesospheric midwinter disturbances: A summary of observed characteristics. J. Geophys. Res., 86, 9665-9678.

Lahoz, W. A., 2000: Northern Hemisphere winter stratospheric variability in the Met. Office Unified Model. Quart. J. Roy. Meteor. Soc., 126, 2605-2630.

Limpasuvan, V., D. W. J. Thompson, and D. L. Hartmann, 2004: The life cycle of the Northern Hemisphere sudden stratospheric warmings. J. Climate, 17, 2584-2596.

Lott, F., and M. J. Miller, 1997: A new subgrid scale orographic drag parameterization: Its formulation and testing. Quart. $J$. Roy. Meteor. Soc., 123, 101-127.

Manzini, E., and L. Bengtsson, 1996: Stratospheric climate and variability from a general circulation model and observations. Climate Dyn., 12, 615-639.

_ , and N. A. McFarlane, 1998: The effect of varying the source spectrum of a gravity wave parameterization in a middle atmosphere general circulation model. J. Geophys. Res., 103, 31 523-31 539.

- - _ and C. McLandress, 1997: Impact of the Doppler Spread Parameterization on the simulation of the middle atmosphere circulation using the MA/ECHAM4 general circulation model. J. Geophys. Res., 102, 25 751-25 762.

— B. B. Steil, C. Brühl, M. A. Giorgetta, and K. Krüger, 2003: A new interactive chemistry-climate model: 2 . Sensitivity of the middle atmosphere to ozone depletion and increase in greenhouse gases and implications for recent stratospheric cooling. J. Geophys. Res., 108, 4429, doi:10.1029/2002JD002977.

Matsuno, T., 1971: A dynamical model of the stratospheric sudden warming. J. Atmos. Sci., 28, 1479-1494.

Pawson, S., et al., 2000: The GCM-Reality Intercomparison Project for SPARC (GRIPS): Scientific issues and initial results. Bull. Amer. Meteor. Soc., 81, 781-796.

Quadrelli, R., and J. M. Wallace, 2002: Dependence of the structure of the Northern Hemisphere annular mode on the polarity of ENSO. Geophys. Res. Lett, 29, 2132, doi:10.1029/ 2002 GL015807.

Ramaswamy, V., and Coauthors, 2001: Stratospheric temperature trends: Observations and model simulations. Rev. Geophys., 39, 71-122.

Randel, W., and Coauthors, 2004: The SPARC intercomparison of middle-atmosphere climatologies. J. Climate, 17, 986-1003.

Roeckner, E., J. M. Oberhuber, A. Bacher, M. Christoph, and I. Kirchner, 1996: ENSO variability and atmospheric response in a global coupled atmosphere-ocean GCM. Climate Dyn., 12, 737-754.

— and Coauthors, 2003: The atmospheric general circulation model ECHAM5. Part I. Model description. MPI Rep. 349, MaxPlanck-Institut für Meteorologie, Hamburg, Germany, 127 pp. — 
horizontal and vertical resolution in the ECHAM5 atmosphere model. J. Climate, 19, 3771-3791.

Rowell, D. P., C. K. Folland, K. Maskell, and M. N. Ward, 1995: Variability of summer rainfall over tropical North Africa (1906-92): Observations and modeling. Quart. J. Roy. Meteor. Soc., 121, 669-704.

Salby, M. L., and P. F. Callaghan, 2003: Systematic changes of stratospheric temperature: Relationship between the tropics and extratropics. J. Geophys. Res., 108, 4101, doi:10.1029/ 2001JD002034.

Sassi, F., D. Kinnison, B. A. Boville, R. R. Garcia, and R. Roble, 2004: Effect of El Niño-Southern Oscillation on the dynamical, thermal, and chemical structure of the middle atmosphere. J. Geophys. Res., 109, D17108, doi:10.1029/ 2003JD004434

Schmidt, H., and Coauthors, 2006: The HAMMONIA chemistry climate model: Sensitivity of the mesopause region to the 11year solar cycle and $\mathrm{CO}_{2}$ doubling. J. Climate, 19, 3903-3931.

Solomon, S., 1999: Stratospheric ozone depletion: A review of concepts and history. Rev. Geophys., 37, 275-316.
Steil, B., C. Brühl, E. Manzini, P. J. Crutzen, J. Lelieveld, P. J. Rasch, E. Roeckner, and K. Krüger, 2003: A new interactive chemistry-climate model: 1 . Present-day climatology and interannual variability of the middle atmosphere using the model and 9 years of HALOE/UARS data. J. Geophys. Res., 108, 4290, doi:10.1029/2002JD002971.

Strauss, D. M., and J. Shukla, 2000: Distinguishing between the SST-forced and internal variability in mid latitudes: Analysis of observations and GCM simulations. Quart. J. Roy. Meteor. Soc., 126, 2323-2350.

- , and - 2002: Does ENSO force the PNA? J. Climate, 15, 2340-2358.

Uppala, S., and Coauthors, 2004: ERA-40: ECMWF 45-year reanalysis of the global atmosphere and surface conditions 1957-2002. ECMWF Newsletter, Vol. 101, ECMWF, Reading, United Kingdom, 2-21.

van Loon, H., and K. Labitzke, 1987: The Southern Oscillation. Part V: The anomalies in the lower stratosphere of the Northern Hemisphere in winter and a comparison with the quasibiennial oscillation. Mon. Wea. Rev., 115, 357-369. 